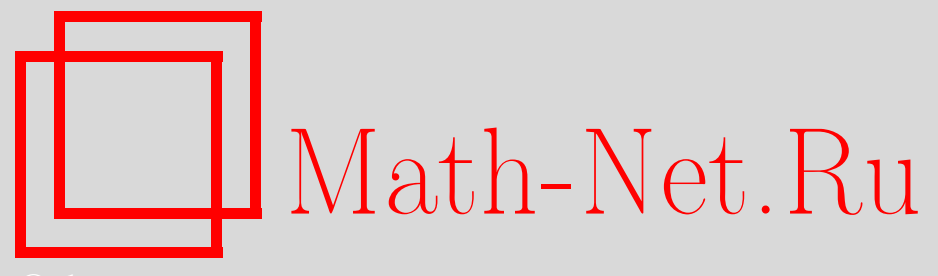

Ю. Л. Павлов, И. А. Чеплюкова, Предельные распределения числа вершин в слоях просто генерируемого леса, Дискрет. матем., 1999, том 11, выпуск 1, 97-112

DOI: https://doi.org/10.4213/dm366

Использование Общероссийского математического портала Math-Net.Ru подразумевает, что вы прочитали и согласны с пользовательским соглашением http://www.mathnet.ru/rus/agreement

Параметры загрузки:

IP: 18.209.158.208

26 апреля 2023 г., 14:43:49 


\title{
Предельные распределения числа вершин в слоях просто генерируемого леса
}

\author{
(C) 1999 г. ..Л. Павлов, И. А. Чеплюкова
}

\begin{abstract}
Рассматриваются просто генерируемые леса, состоящие из $N$ деревьев и содержащие $n$ некорневых вершин. При $N, n \rightarrow \infty$ так, что $N^{2} / n \rightarrow \infty$, получены предельные распределения числа вершин в слоях таких лесов.

Работа выполнена при поддержке Российского фонда фундаментальных исследований, проект 97-01-00065.
\end{abstract}

Задача исследования предельного поведения числа вершин в слоях случайного леса впервые исследовалась, по-видимому, в работах $[1,2]$. Там рассматривались леса, состоящие из $N$ корневых деревьев с помеченными вершинами, корни которых занумерованы числами $1,2, \ldots, N$, а некорневые вершины имеют номера $1,2, \ldots, n$. При этом на множестве всех таких лесов задавалось равномерное распределение вероятностей. Кроме того, в [1] предполагалось, что кратности всех вершин деревьев принимают значения из некоторого подмножества $R$ целых неотрицательных чисел, содержащего ноль (кратность вершины есть число дуг, выходящих из нее при ориентации дуг от корней). Назовем высотой вершины число дуг, образующих путь от корня к этой вершине и множество вершин высоты $t$ назовем $t$-м слоем леca. В [1] были получены предельные распределения числа вершин в $t$-м слое для двух случаев стремления $N$ и $n$ к бесконечности. В первом случае считалось, что $N=c n(1+o(1)), 0<c<1, t \quad$ фиксировано либо $t \rightarrow \infty, n(1-c)^{t} \rightarrow \infty$, а во втором случае $N=O(\sqrt{n}), t / \sqrt{n} \rightarrow 0$. В [2] такие предельные теоремы были доказаны при $n \rightarrow \infty, N / \sqrt{n} \leqslant C_{1}<\infty, t / \sqrt{n} \leqslant C_{2}<\infty$. Заметим, что при этих ограничениях высота случайного леса (максимальный номер слоя) имеет порядок $\sqrt{n}$ (см. [3]), поэтому результаты работы [2] дают исчерпывающую картину предельного поведения числа вершин в слоях леса в рассматриваемой зоне изменения параметров.

Идея доказательства результатов работ $[1,2]$ восходит к известной монографии В. Ф. Колчина [4], где рассматривалась асимптотика (при $n \rightarrow \infty$ ) числа вершин в слоях случайного дерева с $n$ помеченными вершинами. В [4] эта задача сводилась к рассмотрению критического ветвящегося процесса Гальтона-Ватсона, начинающегося с одной частицы, с пуассоновским распределением числа прямых потомков каждой частицы при условии, что общее число частиц, существовавших в процессе до его вырождения, равно $n$. Естественно, что при изучении описанных выше случайных лесов в $[1,2]$ использовался аналогичный ветвящийся процесс, начинающийся с $N$ частиц. 
В [5] были получены результаты, аналогичные [2], для лесов, состоящих из $N$ плоских деревьев с висячими корнями и $n$ некорневыми вершинами при равномерном распределении вероятностей на множестве таких лесов. В этом случае для доказательства предельных теорем использовался условный ветвящийся процесс Гальтона-Ватсона с геометрическим распределением числа потомков каждой частицы.

В статьях $[6,7]$ расматривались леса, состоящие из $N$ просто генерируемых деревьев. Определение просто генерируемого семейства корневых деревьев дано в [8]. Известно (см., например, [9]), что каждое конкретное просто генерируемое семейство можно рассматривать как множество реализаций некоторого ветвящегося процесса Гальтона-Ватсона, начинающегося с одной частицы. Аналогично, ветвящийся процесс $G$, начинающийся с $N$ частиц, образует просто генерируемый лес из $N$ деревьев и естественным образом индуцирует на нем распределение вероятностей. Обозначим $\mathscr{F}_{N, n}$ совокупность таких лесов, содержащих $n$ некорневых вершин. Из $[8,9]$ следует, что случайные леса, рассматривавашиеся в $[1,2,3,5]$ являются частными случаями $\mathscr{F}_{N, n}$. Пусть $\chi$ - некоторая числовая характеристика леса из $\mathscr{F}_{N, n}, \chi(G)$ - аналогичная характеристика соответствующего ветвящегося процесса $G$. Пусть также $\nu_{N}$ обозначает случайную величину, равную числу частиц процесса $G$ за все время его эволюции. В [7] доказано следующее утверждение.

Лемма 1. Справедливо равенство

$$
\mathbf{P}\{\chi=x\}=\mathbf{P}\left\{\chi(G)=x \mid \nu_{N}=N+n\right\} .
$$

Эта лемма показывает, что изучение числовых характеристик просто генерируемого леса можно свести к исследованию аналогичных характеристик ветвящегося процесса $G$ при условии $\nu_{N}=N+n$. Лемма 1 является обобщением леммы 1.4.1 книги [3], поэтому все результаты о случайных лесах, полученные в [3], распространяются и на $\mathscr{F}_{N, n}$.

Обозначим $\mu\left(t, \mathscr{F}_{N, n}\right)$ число вершин в $t$-м слое леса из $\mathscr{F}_{N, n}$, а $\mu_{N}(t)$ - число частиц $t$-го поколения ветвящегося процесса $G$. Из леммы 1 следует, что распределения $\mu\left(t, G_{N, n}\right)$ и $\mu_{N}(t)$ связаны следующим образом.

Лемма 2. При любых $x$ u $t$ справедливо равенство

$$
\mathbf{P}\left\{\mu\left(t, \mathscr{F}_{N, n}\right)=x\right\}=\mathbf{P}\left\{\mu_{N}(t)=x \mid \nu_{N}=N+n\right\}
$$

Для случая существования конечной дисперсии числа потомков частиц процесса $G$ в [6] получено полное описание предельного поведения $\mu\left(t, \mathscr{F}_{N, n}\right)$ при $n \rightarrow \infty$, $N / \sqrt{n} \leqslant C_{1}<\infty$. В данной статье рассматривается асимптотика этой случайной величины в других зонах изменения параметров $N, n$. При этом номера слоев $t$ в полученных ниже результатах, грубо говоря, удовлетворяют соотношению $t=o(\tau)$, где $\tau$ - высота леса (предельные распределения высоты леса во всех случаях стремления $N, n$ к бесконечности получены в [3]).

Введем вспомогательную случайную величину $\xi$, имеющую распределение

$$
\mathbf{P}\{\xi=k\}=p_{k}, \quad k=0,1,2, \ldots
$$

с максимальным шагом $d$ и производящей функцией

$$
F(z)=\sum_{k=0}^{\infty} p_{k} z^{k}
$$


причем множество значений $\xi$, имеющих ненулевую вероятность, содержит ноль и не совпадает с множеством $\{0,1\}$. Кроме того, мы будем считать, что $\mathbf{M} \xi=1$, $\mathbf{D} \xi=B<\infty, F^{\prime \prime \prime}(1)<\infty$.

Известно (см., например, [9]), что если конечны первые три момента распределения числа прямых потомков частиц генерирующего $\mathscr{F}_{N, n}$ ветвящегося процесса $G$, то это распределение можно задать следующим образом:

$$
p_{k}(\lambda)=\lambda^{k} p_{k} / F(\lambda), \quad k=0,1,2, \ldots, \quad \lambda>0 .
$$

Далее мы будем полагать, что параметр $\lambda=\lambda(N, n)$ определяется соотношением

$$
\frac{\lambda F^{\prime}(\lambda)}{F(\lambda)}=\frac{n}{N+n} \text {. }
$$

Обозначим $m$ и $B_{\lambda}$ соответственно математическое ожидание и дисперсию распределения (1). Из (2) следует, что $m=n /(N+n)$.

Справедливы следующие результаты.

Теорема 1. Пусть $N, n \rightarrow \infty$ так, ито $n / N \leqslant C<\infty, N m^{t} \rightarrow \infty$. Пусть также $t \geqslant 3$ при $n / N \rightarrow 0$ и $t \rightarrow \infty$ в противном случае. Тогда равномерно относителъно $k$, для которых

$$
y=\left(k-N m^{t}\right) / \sqrt{(N+n) B_{\lambda} m^{t-1}}
$$

лежит в любом конечном интервале,

$$
\mathbf{P}\left\{\mu\left(t, \mathscr{F}_{N, n}\right)=k\right\}=\frac{d(1+o(1))}{\sqrt{2 \pi(N+n) B_{\lambda} m^{t-1}}} e^{-y^{2} / 2} .
$$

Теорема 2. Пусть $N, n \rightarrow \infty$ так, что $n / N \rightarrow \infty, n / N^{2} \rightarrow 0$, a $t=t(N, n)$ выбрано так, что $t N /\left(n \ln \left(N^{2} / n\right)\right) \rightarrow 0, t N / n \rightarrow \infty$ и для некоторого $L>0$ при достаточно болъших $N, n$ справедливо неравенство $\max \{n / N, N / \sqrt{n}\} \leqslant\left(N \sqrt{m^{t} / n}\right)^{L}$. Тогда равномерно относителъно $k$, для которых $y=\left(k-N m^{t}\right) / \sqrt{B n m^{t}}$ лежит в любом конечном интервале,

$$
\mathbf{P}\left\{\mu\left(t, \mathscr{F}_{N, n}\right)=k\right\}=\frac{d(1+o(1))}{\sqrt{2 \pi B n m^{t}}} e^{-y^{2} / 2} .
$$

Теорема 3. Пусть $N, n \rightarrow \infty$ так, что $n / N \rightarrow \infty, n / N^{2} \rightarrow 0$, a $t=t(N, n)$ выбрано так, что $t N / n \rightarrow \alpha, 0<\alpha<\infty$ и для некоторого $L>0$ при достаточно больиих $N, n$ выполняется неравенство $n / N<(N / \sqrt{n})^{L}$. Тогда равномерно относителъно $k$, для которых $y=\left(k-N m^{t}\right) / \sqrt{H B n}$ лежит в любом конечном интервале,

$$
\mathbf{P}\left\{\mu\left(t, \mathscr{F}_{N, n}\right)=k\right\}=\frac{d(1+o(1))}{\sqrt{2 \pi H B n}} e^{-y^{2} / 2},
$$

¿de

$$
\begin{gathered}
H=e^{-2 \alpha}\left(1-e^{-\alpha}\right)\left(1+Q\left(1-\rho^{2}\right) e^{\alpha}\right) \\
Q=1-2 \alpha e^{-\alpha}-e^{-2 \alpha}, \quad \rho=\frac{\left(\alpha-1+e^{-\alpha}\right) e^{-\alpha / 2}}{\sqrt{\left(1-e^{-\alpha}\right) Q}}
\end{gathered}
$$


Теорема 4. Пустъ $N, n \rightarrow \infty \operatorname{max,~что~} n / N \rightarrow \infty, n / N^{2} \rightarrow 0$, a $t=t(N, n)$ въбрано так, что $t N / n \rightarrow 0, N^{4} t^{2} / n^{3} \geqslant C>0$ u для некоторого $L>0$ при достаточно больших $N, n$ выполняется неравенство $n / N<(N / \sqrt{n})^{L}$. Тогда равномерно относителъно $k$, для которых $y=\left(k-N m^{t}\right) / \sqrt{N B t}$ лежит в любом конечном интервале,

$$
\mathbf{P}\left\{\mu\left(t, \mathscr{F}_{N, n}^{\prime}\right)=k\right\}=\frac{d(1+o(1))}{\sqrt{2 \pi N B t}} e^{-y^{2} / 2} .
$$

Теорема 5. Пусть $N, n \rightarrow \infty$ maк, что $n / N \rightarrow \infty, n / N^{2} \rightarrow 0, N^{4} t^{2} / n^{3} \rightarrow 0$. Тогда для любого фиксированного $x$

$$
\mathbf{P}\left\{\frac{\mu\left(t, \mathscr{F}_{N, n}\right)-N m^{t}}{\sqrt{B N t}} \leqslant x\right\} \rightarrow \frac{1}{\sqrt{2 \pi}} \int_{-\infty}^{x} e^{-z^{2} / 2} d z .
$$

Теорема 6. Пусть $N, n \rightarrow \infty$ maк, что $0<C_{1} \leqslant n / N \leqslant C_{2}<\infty, t \geqslant 1$ биксировано. Тогда равномерно относителъно целых $k_{i}, i=1, \ldots, t, \operatorname{maких,~что~} u_{i}=$ $\left(k_{i}-N m^{i}\right) / \sqrt{(N+n) B_{\lambda}}$ лежат в любых конечных интервалах,

$$
\begin{aligned}
\mathbf{P}\left\{\mu\left(1, \mathscr{F}_{N, n}\right)\right. & \left.=k_{1}, \ldots, \mu\left(t, \mathscr{F}_{N, n}\right)=k_{t}\right\} \\
& =\left(2 \pi(N+n) B_{\lambda} d^{-2}\right)^{-t / 2} \sqrt{\operatorname{det} Q(m)} \exp \left\{-2^{-1} u Q(m) u^{\prime}\right\}(1+o(1)),
\end{aligned}
$$

где $u=\left(u_{1}, \ldots, u_{t}\right), Q(m)=\left\|q_{i j}(m)\right\|_{i, j=1}^{t}$ - матрица со следующими элементами:

$$
\begin{gathered}
q_{i i}(m)= \begin{cases}\left((1-m)^{3}+m^{t+1-i}(1+m)\right) / m^{t}(1-m), & 1 \leqslant i \leqslant t-1 ; \\
1 / m^{t}(1-m), & i=t ;\end{cases} \\
q_{i j}(m)=q_{j i}(m)= \begin{cases}\left((1-m)^{3}-m^{t+1-i}\right) / m^{t}(1-m), & 1 \leqslant i \leqslant t-2, j=i+1 ; \\
(1-m)^{3} / m^{t}(1-m), & 1 \leqslant i \leqslant t-3, i+2 \leqslant j \leqslant t-1 ; \\
(1-m)^{2} / m^{t}(1-m), & 1 \leqslant i \leqslant t-2, j=t ; \\
(1-2 m) / m^{t}(1-m), & i=t-1, j=t .\end{cases}
\end{gathered}
$$

Теоремы 1-6 являются обобщением результатов работы [1] о случайных лесах на просто генерируемые леса и различные зоны изменения параметров $N, n, t$. Для их доказательства оказалось достаточно применить идеи и методы, использованные в [1]. В то же время следует отметить, что при доказательстве теорем 1-6 этим путем возникает необходимость проведения хотя и элементарных, но весьма громоздких по сравнению с [1] выкладок, что и является основной трудностью получения этих результатов.

Далее приводятся вспомогательные утверждения (леммы 3-14), а затем с их помощью доказываются теоремы 1-6.

Легко видеть, что ветвящийся процесс $G$ естественным образом распадается на $N$ независимых процессов $G_{1}, \ldots, G_{N}$, каждый из которых начинается с одной частицы. Тогда $\mu_{1}(t)$ и $\nu_{1}$ есть, соответственно, число частиц $t$-го поколения и общее число частиц процесса $G_{1}$. Обозначим $\nu_{N}(t)=\mu_{N}(0)+\ldots+\mu_{N}(t-1), t=1,2, \ldots$, $\nu_{N}(0)=0$, следовательно, $\nu_{1}(t)$ есть аналогичная характеристика $G_{1}$. Для доқазательства теорем 1-4 нам потребуется рассмотреть пять различных случаев изменения параметров $N, n, t$ при $N, n \rightarrow \infty, n / N^{2} \rightarrow 0$. Введем условия А1-A5:

$$
n / N \rightarrow 0, N m^{t} \rightarrow \infty, t \geqslant 3
$$


(A2) $0<C_{1} \leqslant n / N \leqslant C_{2}<\infty, N m^{t} \rightarrow \infty, t \rightarrow \infty$;

(A3) $n / N \rightarrow \infty, t N / n \rightarrow \infty, t N /\left(n \ln N^{2} / n\right) \rightarrow 0$

(A4) $n / N \rightarrow \infty, t N / n \rightarrow \alpha, 0<\alpha<\infty$;

(A5) $n / N \rightarrow \infty, t N / n \rightarrow 0, N^{4} t^{2} / n^{3} \geqslant C>0$.

Далее нам потребуется изучить асимптотику совместного распределения $\mu_{N}(t)$ и $\nu_{N}(t)$. Для этого рассмотрим сначала поведение характеристической функции $\Psi_{t}\left(\theta_{1}, \theta_{2}\right)$ случайного вектора $\left(\mu_{1}(t)-\mathbf{M} \mu_{1}(t), \nu_{1}(t)-\mathbf{M} \nu_{1}(t)\right)$.

Лемма 3. Пустъ выполнено одно из условий А1, А2, А3, А5. Тогда для любых фиксированных $\theta_{1} \quad u \theta_{2}$

$$
\Psi_{t}^{N}\left(\frac{\theta_{1}}{\sqrt{N \mathrm{D} \mu_{1}(t)}}, \frac{\theta_{2}}{\sqrt{N \mathrm{D} \nu_{1}(t)}}\right)=\exp \left\{-\frac{1}{2}\left(\theta_{1}^{2}+\theta_{2}^{2}\right)\right\}(1+o(1)) .
$$

Доказательство. Нетрудно проверить, что для $\Psi_{t}\left(\theta_{1}, \theta_{2}\right)$ справедливо разложение

$$
\Psi_{t}\left(\theta_{1}, \theta_{2}\right)=1-2^{-1} \theta_{1}^{2} \mathbf{D} \mu_{1}(t)-2^{-1} \theta_{2}^{2} \mathbf{D} \nu_{1}(t)-\theta_{1} \theta_{2} \operatorname{cov}\left(\mu_{1}(t), \nu_{1}(t)\right)+\varepsilon\left(\theta_{1}, \theta_{2}\right),
$$

где

$$
\begin{aligned}
\mathbf{M} \mu_{1}(t) & =m^{t} \\
\mathbf{D} \mu_{1}(t) & =B_{\lambda} m^{t-1}\left(1-m^{t}\right)(1-m)^{-1} \\
\mathbf{M} \nu_{1}(t) & =\left(1-m^{t}\right) /(1-m) \\
\mathbf{D} \nu_{1}(t) & =B_{\lambda}\left(1-(2 t-1)(1-m) m^{t-1}-m^{2 t-1}\right)(1-m)^{-3} \\
\operatorname{cov}\left(\mu_{1}(t), \nu_{1}(t)\right) & =B_{\lambda} m^{t-1}\left((1-m) t-\left(1-m^{t}\right)\right)(1-m)^{-2} \\
\left|\varepsilon\left(\theta_{1}, \theta_{2}\right)\right| & \leqslant C_{3}\left(\left|\theta_{1}\right|^{3} \mathbf{M} \mu_{1}^{3}(t)+\left|\theta_{2}\right|^{3} \mathbf{M} \nu_{1}^{3}(t)\right)
\end{aligned}
$$

$C_{3}$ - некоторая положительная постоянная. Используя явные выражения $\mathbf{M} \mu_{1}^{3}(t)$ и $\mathrm{M} \nu_{1}^{3}(t)$ во всех рассматриваемых случаях, а также соотношения (3), (4), приходим к утверждению леммы 3 .

Аналогично можно доказать следующий результат.

Лемма 4. При выполнении условия А4 для любых фиксированных $\theta_{1} u \theta_{2}$

$$
\Psi_{t}^{N}\left(\frac{\theta_{1}}{\sqrt{N \mathbf{D} \mu_{1}(t)}}, \frac{\theta_{2}}{\sqrt{N \mathrm{D} \nu_{1}(t)}}\right)=\exp \left\{-\frac{1}{2}\left(\theta_{1}^{2}+\theta_{2}^{2}\right)-\theta_{1} \theta_{2} \rho\right\}(1+o(1))
$$

где величина $\rho$ определена в теореме 3.

Лемма 5. Пусть выполнено одно из условий А1-А5. Тогда при достаточно больших $N$, $n$ найдутся такие положителъные постоянные $\delta_{1}, C$, что если $\left|\theta_{1}\right| \leqslant \delta_{1}$, $\left|\theta_{2}\right| \leqslant \delta_{1}$ в случаях A1, A2, $\left|\theta_{1}\right| \leqslant \delta_{1}(N / n),\left|\theta_{2}\right| \leqslant \delta_{1}(N / n)^{2}$ в случаях A3, A4 u $\left|\theta_{1}\right| \leqslant \delta_{1} / t,\left|\theta_{2}\right| \leqslant \delta_{1} N / n t$ в случае A5, то справедливо неравенство

$$
\left|\Psi_{t}\left(\theta_{1}, \theta_{2}\right)\right| \leqslant \exp \left\{-C u\left(N, n, \theta_{1}, \theta_{2}\right)\right\}
$$


где при выполнении условий $\mathrm{A} 1$ и $\mathrm{A} 2$

$$
u\left(N, n, \theta_{1}, \theta_{2}\right)=\theta_{1}^{2} m^{t}+\theta_{2}^{2} m,
$$

при выполнении условий А3 и А4

$$
u\left(N, n, \theta_{1}, \theta_{2}\right)=\theta_{1}^{2} m^{t}+\theta_{2}^{2}(n / N)^{2},
$$

а при условии А5

$$
u\left(N, n, \theta_{1}, \theta_{2}\right)=t\left(\theta_{1}^{2}+\theta_{2}^{2} t^{2}\right)
$$

Доказателъство. Все утверждения леммы 5 следуют непосредственно из (3) и (4). В случае выполнения условий $\mathrm{A} 1$ и $\mathrm{A} 2$ при этом возникают оценки

$$
\begin{gathered}
\mathbf{D} \mu_{1}(t) \geqslant C_{3} m^{t}, \quad \mathbf{D} \nu_{1}(t) \geqslant C_{4} m, \quad\left|\operatorname{cov}\left(\mu_{1}(t), \nu_{1}(t)\right)\right| \leqslant C_{5} t m, \\
\left|\Psi_{t}\left(\theta_{1}, \theta_{2}\right)\right| \leqslant 1-2^{-1} C_{4}\left(\theta_{1}^{2} m^{t}+\theta_{2}^{2}\right)+m^{(t+1) / 2}\left|\theta_{1} \theta_{2}\right|\left(-C_{4}+C_{3} t m^{t / 2}\right),
\end{gathered}
$$

где $C_{3}, C_{4}, C_{5}$ - некоторые положительные постоянные, а при достаточно больших $N, n$ выполняется неравенство $-C_{4}+C_{3} t m^{t / 2}<0$. Аналогично в случае А3 получаем, что

$\left|\Psi_{t}\left(\theta_{1}, \theta_{2}\right)\right| \leqslant 1-2^{-1} C_{4}(n / N)\left(\theta_{1}^{2} m^{t}+\theta_{2}^{2}(n / N)^{2}\right)+m^{t / 2}(n / N)\left|\theta_{1} \theta_{2}\right|\left(-C_{4}(n / N)+C_{3} t m^{t / 2}\right)$

с отрицательным последним слагаемым. Если же выполнено условие А4, то

$$
\left|\Psi_{t}\left(\theta_{1}, \theta_{2}\right)\right| \leqslant 1-2^{-1} C_{4}(n / N)\left(\theta_{1}^{2} \theta_{2}^{2}(n / N)^{2}\right)+(n / N)^{2}\left|\theta_{1} \theta_{2}\right|\left(-C_{4}+C_{3}\right),
$$

где при достаточно больших $N, n$ постоянные $C_{3}$ и $C_{4}$ можно выбрать такими, что $-C_{4}+C_{3}<0$. Аналогично рассматривается и случай $\mathrm{A} 5$.

Обозначим $f(z)$ производящую функцию случайной величины $\nu_{1}$, т.е.

$$
f(z)=\sum_{k=0}^{\infty} \cdot \mathbf{P}\left\{\nu_{1}=k\right\} z^{k} .
$$

Лемма 6. Пусть $z \rightarrow 1,|z| \leqslant 1, m \rightarrow 1$. Тогда

$$
f(z)=1-\frac{2 \sqrt{1-z}}{\sqrt{2 B+w^{2}}+w}(1+o(1))
$$

где $w=(1-z m) / \sqrt{1-z}$.

Заметим, что в критическом случае $m=1, w \rightarrow 0$ и соответствующий результат доказан в [4] (лемма 2.4.4). Доказательство леммы 6 для случая $m \rightarrow 1, w \rightarrow 0$ приводится в [3] (лемма 1.3.3), оно сохраняет силу и при $w \not A 0$.

Лемма 7. Пусть $\left|\theta_{1}\right| \leqslant \pi / d,\left|\theta_{2}\right| \geqslant 4 m^{t / 2} \sqrt{\mathrm{D} \nu_{1}}, u$ выполнено одно из условий $\mathrm{A} 1$, A2, А3. Тогда при достаточно болъших $N, n$ найдется такое положителъное $\delta_{2}$, что при $\left|\theta_{2}\right| \leqslant \delta_{2}$ в случаях A1, A2 u $\left|\theta_{2}\right| \leqslant \delta_{2}(N / n)^{2}$ в случае A3

$$
\left|\Psi_{t}\left(\theta_{1}, \theta_{2}\right)\right| \leqslant \exp \left\{-\mathbf{D} \nu_{1} \theta_{2}^{2} / 8\right\} \text {. }
$$


Доказателъство. Обозначим $f_{t}(y, z)$ производящую функцию случайного вектора $\left(\mu_{1}(t), \nu_{1}(t)\right)$. В [1] показано, что

$$
f(z)-f_{t}(y, z)=z\left(f(z)-f_{t-1}(y, z)\right) \sum_{r=1}^{\infty} p_{r}(\lambda) \sum_{k=1}^{r} f^{r-k}(z) f_{t-1}^{k-1}(y, z),
$$

откуда следует, что

$$
\left|f_{t}(y, z)\right| \leqslant|f(z)|+2 m^{t}
$$

Нетрудно убедиться, применяя (4), что $\left|f\left(e^{i \theta_{2}}\right)\right| \leqslant 1-\mathbf{D} \nu_{1} \theta_{2}^{2} / 4$, поэтому из соотношения $m^{t} \rightarrow 0$ и (6) следует утверждение леммы 7 .

Лемма 8. Пусть выполнено одно из условий А1-А5. Тогда при достаточно больших $N, n$ для любого $\delta>0$ найдется такое $q_{0}<1$, что при $\delta \leqslant\left|\theta_{2}\right| \leqslant \pi / d,\left|\theta_{1}\right| \leqslant \pi / d$

$$
\left|\Psi_{t}\left(\theta_{1}, \theta_{2}\right)\right| \leqslant q_{0}
$$

Доказателъство. Легко видеть, что максимальный шаг распределения $\nu_{1}$ равен $d$, поэтому для любого $\delta>0$ найдется такое $q<1$, что при $\delta \leqslant\left|\theta_{2}\right| \leqslant \pi / d$ справедливо неравенство

$$
\left|f\left(e^{i \theta_{2}}\right)\right| \leqslant q .
$$

Пусть выполнено одно из условий А1-А3. Тогда $m^{t} \rightarrow 0$ и утверждение леммы 8 следует из (6) и (7). Рассмотрим условия А4 и А5. Раскрывая суммы, стоящие в правой части равенства (5), получаем, что

$$
\left|\sum_{r=1}^{\infty} p_{r}(\lambda) \sum_{k=1}^{r} f^{r-k}\left(e^{i \theta_{2}}\right) f_{t-1}^{k-1}\left(e^{i \theta_{1}}, e^{i \theta_{2}}\right)\right| \leqslant(1-q)^{-1}\left(1-p_{0}(\lambda)-q p_{1}(\lambda)-q^{2} p_{2}(\lambda)-\ldots\right) .
$$

Используя явный вид вероятностей $p_{0}(\lambda), p_{1}(\lambda), \ldots$ (см. (1)) и свойства производящей функции $F(z)$, нетрудно показать, что

$$
\left|\sum_{r=1}^{\infty} p_{r}(\lambda) \sum_{k=1}^{r} f^{r-k}\left(e^{i \theta_{2}}\right) f_{t-1}^{k-1}\left(e^{i \theta_{1}}, e^{i \theta_{2}}\right)\right| \leqslant q_{1}<1 .
$$

Отсюда и из (5) следует, что при $t \rightarrow \infty$ выполняется соотношение

$$
\left|f\left(e^{i \theta_{2}}\right)-f_{t}\left(e^{i \theta_{1}}, e^{i \theta_{2}}\right)\right| \leqslant q_{1}^{t} \rightarrow 0,
$$

поэтому из (7) следует лемма 8 и для этого случая.

Лемма 9. Пустъ выполнено одно из условий А1-А5. Тогда при достаточно больших $N, n$ для любого $\delta>0$ найдется такая положительная постоянная $C_{3}$, что при $\delta N / n \leqslant\left|\theta_{1}\right| \leqslant \pi / d,\left|\theta_{2}\right| \leqslant \pi / d$ в случаях $\mathrm{A} 1-\mathrm{A} 4$

$$
\left|\Psi_{t}\left(\theta_{1}, \theta_{2}\right)\right| \leqslant \exp \left\{-C_{3}\left|\theta_{1}\right| m^{t} N / n\right\},
$$

а в случае А5

$$
\left|\Psi_{t}\left(\theta_{1}, \theta_{2}\right)\right| \leqslant \exp \left\{-C_{3}\left|\theta_{1}\right| / t\right\} .
$$


Доказательство. В [1] показано, что

$$
\left|f_{t}(y, z)\right| \leqslant 1-(1-|F(y)|) \mathbf{P}\left\{\mu_{1}(t-1) \geqslant 1\right\} .
$$

Легко видеть, что если $\left|\theta_{1}\right| \geqslant \delta$, то существует положительная постоянная $q<1$ такая, что $\left|F\left(e^{i \theta_{1}}\right)\right|<q$, а если $\delta N / n \leqslant\left|\theta_{1}\right|<\delta$, то существует такое $C>0$, что $\left|F\left(e^{i \theta_{1}}\right)\right| \leqslant 1-C\left|\theta_{1}\right|$. Согласно теореме 2.2.2 книги [10]

$$
\mathbf{P}\left\{\mu_{1}(t) \geqslant 1\right\}=K m^{t}(1+o(1)), \quad 0<K<\infty,
$$

поэтому утверждение леммы справедливо при выполнении условия А2. B случае A1 оно следует из (9), (10) и следствия 1.3.1 [3], где показано, что $K=1$. Применяя в оставшихся случаях теорему 1.3.4 [3], получаем, что

$$
\mathbf{P}\left\{\mu_{1}(t) \geqslant 1\right\}=m^{t} N / n(1+o(1))
$$

для условий $\mathrm{A} 3, \mathrm{~A} 4$ и

$$
\mathbf{P}\left\{\mu_{1}(t) \geqslant 1\right\}=2 / B t(1+o(1))
$$

для условия $\mathrm{A} 5$, поэтому утверждение леммы 9 следует из (9).

Лемма 10. Пусть выполнено одно из условий А3, А4, А5. Тогда при достаточно больших $N, n$ для любого $\delta>0$ найдется такая положительная постоянная $C_{4}$, что при $\delta(N / n)^{2} \leqslant\left|\theta_{2}\right| \leqslant \delta,\left|\theta_{1}\right| \leqslant \pi / d$ в случалх $\mathrm{A} 3$ и $\mathrm{A} 4$

$$
\left|\Psi_{t}\left(\theta_{1}, \theta_{2}\right)\right| \leqslant \exp \left\{-C_{4} \sqrt{\left|\theta_{2}\right|}\right\}
$$

а в случае A5

$$
\left|\Psi_{t}\left(\theta_{1}, \theta_{2}\right)\right| \leqslant \exp \left\{-C_{4} t\left|\theta_{2}\right|\right\} .
$$

Доказательство. Из (5) следует, что

$$
\left|f(z)-f_{t}(y, z)\right| \leqslant\left|f(z)-f_{t-1}(y, z)\right| \sum_{r=1}^{\infty} r p_{r}(\lambda)=m\left|f(z)-f_{t-1}(y, z)\right|,
$$

поэтому

$$
\left|f(z)-f_{t}(y, z)\right| \leqslant m^{t}\left|f(z)-f_{1}(y, z)\right|,
$$

при этом $f_{1}(y, z)=z F(y)$ [1]. Используя лемму 6 и то, что в условиях $\mathrm{A} 3 m^{t} \rightarrow 0$, а при $\mathrm{A} 4 m^{t} \rightarrow e^{-\alpha}<1$, получаем требуемое утверждение для этих двух случаев. Пусть выполнено условие А5. Рассуждая аналогично тому, как была получена оценка (8), нетрудно доказать, что при достаточно больших $N, n$ существует такое $C_{5}>0$, что

$$
\left|\sum_{r=1}^{\infty} p_{r}(\lambda) \sum_{k=1}^{r} f^{r-k}\left(e^{i \theta_{2}}\right) f_{t-1}^{k-1}\left(e^{i \theta_{1}}, e^{i \theta_{2}}\right)\right| \leqslant \exp \left\{-C_{5}\left(1-\left|f\left(e^{i \theta_{2}}\right)\right|-N / n\right)\right\} .
$$

Применяя лемму 6, отсюда получаем, что последнее выражение не превосходит $\exp \left\{-C_{6} \sqrt{\left|\theta_{2}\right|}\right\}$, где $C_{6}$ - некоторая положительная постоянная. Отсюда и из (5) следует, что

$$
\left|f\left(e^{i \theta_{2}}\right)-f_{t}\left(e^{i \theta_{1}}, e^{i \theta_{2}}\right)\right| \leqslant\left|f\left(e^{i \theta_{2}}\right)-f_{1}\left(e^{i \theta_{1}}, e^{i \theta_{2}}\right)\right| \exp \left\{-C_{6} t \sqrt{\left|\theta_{2}\right|}\right\} .
$$

Опять используя лемму 6 и соотношение $f_{1}(y, z)=z F(y)$, приходим к утверждению леммы 10 в случае $\mathrm{A} 5$. 
Лемма 11. При выполнении условий любой из теорем $1,2,4$

$$
\sup _{k, s}\left|N d^{-2} \sqrt{\mathbf{D} \mu_{1}(t) \mathbf{D} \nu_{1}(t)} \mathbf{P}\left\{\mu_{N}(t)=k, \nu_{N}(t)=s\right\}-(2 \pi)^{-1} \exp \left\{-\left(u^{2}+v^{2}\right) / 2\right\}\right| \rightarrow 0
$$

əde

$$
u=\left(k-N \mathrm{M} \mu_{1}(t)\right) / \sqrt{N \mathrm{D} \mu_{1}(t)}, \quad v=\left(s-N \mathrm{M} \nu_{1}(t)\right) / \sqrt{N \mathrm{D} \nu_{1}(t)} .
$$

Дожазательство. Утверждение леммы 11 можно получить, следуя обычной схеме доказательства локальных предельных теорем. Для этого достаточно показать, что разность

$$
R(u, v)=4 \pi^{2} d^{-2} N \sqrt{\mathbf{D} \mu_{1}(t) \mathbf{D} \nu_{1}(t)} \mathbf{P}\left\{\mu_{N}(t)=k, \nu_{N}(t)=s\right\}-2 \pi \exp \left\{-\left(u^{2}+v^{2}\right) / 2\right\}
$$

стремится к нулю. По формулам обращения

$$
\begin{aligned}
& \frac{1}{2 \pi} \exp \left\{-\frac{1}{2}\left(u^{2}+v^{2}\right)\right\}=\frac{1}{4 \pi^{2}} \int_{-\infty}^{\infty} \int_{-\infty}^{\infty} \exp \left\{-i \theta_{1} u-i \theta_{2} v-\frac{1}{2}\left(\theta_{1}^{2}+\theta_{2}^{2}\right)\right\} d \theta_{1} d \theta_{2}, \\
& \begin{aligned}
\mathbf{P}\left\{\mu_{N}(t)=\right. & \left.k, \nu_{N}(t)=s\right\} \\
& =d^{2}\left(4 \pi N \sqrt{\mathbf{D} \mu_{1}(t) \mathbf{D} \nu_{1}(t)}\right)^{-1} \iint \exp \left\{-i \theta_{1} u-i \theta_{2} v\right\} \varphi_{N, t}\left(\theta_{1}, \theta_{2}\right) d \theta_{1} d \theta_{2}
\end{aligned}
\end{aligned}
$$

где

$$
\varphi_{N, t}\left(\theta_{1}, \theta_{2}\right)=\Psi_{t}^{N}\left(\frac{\theta_{1}}{\sqrt{N \mathrm{D} \mu_{1}(t)}}, \frac{\theta_{2}}{\sqrt{N \mathrm{D} \nu_{1}(t)}}\right)
$$

а интегрирование проводится по области вида

$$
\left|\theta_{1}\right| \leqslant \pi \sqrt{N \mathbf{D} \mu_{1}(t)} / d, \quad\left|\theta_{2}\right| \leqslant \pi \sqrt{N \mathbf{D} \nu_{1}(t)} / d .
$$

Следуя [1], обозначим

$$
\begin{aligned}
& M_{1}=\left\{\left(\theta_{1}, \theta_{2}\right):\left|\theta_{1}\right| \leqslant T,\left|\theta_{2}\right| \leqslant T\right\} \\
& M_{2}=\left\{\left(\theta_{1}, \theta_{2}\right):\left|\theta_{1}\right| \leqslant \delta \sqrt{N \mathbf{D} \mu_{1}(t)}, T<\left|\theta_{2}\right| \leqslant \delta \sqrt{N \mathrm{D} \nu_{1}(t)}\right\}, \\
& M_{3}=\left\{\left(\theta_{1}, \theta_{2}\right): T<\left|\theta_{1}\right| \leqslant \delta \sqrt{N \mathrm{D} \mu_{1}(t)},\left|\theta_{2}\right| \leqslant T\right\}, \\
& M_{4}=\left\{\left(\theta_{1}, \theta_{2}\right): \delta \sqrt{N \mathrm{D} \mu_{1}(t)}<\left|\theta_{1}\right| \leqslant \pi \sqrt{N \mathrm{D} \mu_{1}(t)} / d,\left|\theta_{2}\right| \leqslant 4 \sqrt{N m^{t}}\right\}, \\
& M_{5}=\left\{\left(\theta_{1}, \theta_{2}\right): \delta \sqrt{N \mathrm{D} \mu_{1}(t)}<\left|\theta_{1}\right| \leqslant \pi \sqrt{N \mathbf{D} \mu_{1}(t)} / d, 4 \sqrt{N m^{t}}<\left|\theta_{2}\right| \leqslant \delta \sqrt{N \mathrm{D} \nu_{1}(t)}\right\}, \\
& M_{6}=\left\{\left(\theta_{1}, \theta_{2}\right):\left|\theta_{1}\right| \leqslant \pi \sqrt{N \mathrm{D} \mu_{1}(t)} / d, \delta \sqrt{N \mathrm{D} \nu_{1}(t)}<\left|\theta_{2}\right| \leqslant \pi \sqrt{N \mathrm{D} \nu_{1}(t)} / d\right\},
\end{aligned}
$$

где выбор положительных постоянных $\delta, T$ ясен из дальнейшего. Легко видеть, что разность $R(u, v)$ можно представить в виде суммы интегралов

$$
R(u, v)=\sum_{j=1}^{8} I_{j}
$$


где

$$
\begin{aligned}
& I_{1}=\iint_{M_{1}} \exp \left\{-i \theta_{1} u-i \theta_{2} v\right\}\left(\varphi_{N, t}\left(\theta_{1}, \theta_{2}\right)-\exp \left\{-2^{-1}\left(\theta_{1}^{2}+\theta_{2}^{2}\right)\right\}\right) d \theta_{1} d \theta_{2} \\
& I_{j}=\iint_{M_{j}} \exp \left\{-i \theta_{1} u-i \theta_{2} v\right\} \varphi_{N, t}\left(\theta_{1}, \theta_{2}\right) d \theta_{1} d \theta_{2}, \quad j=2,3, \ldots 6 \\
& I_{7}=-\int_{-\infty}^{\infty} \int_{\left|\theta_{2}\right| \geqslant T} \exp \left\{-i \theta_{1} u-i \theta_{2} v-2^{-1}\left(\theta_{1}^{2}+\theta_{2}^{2}\right)\right\} d \theta_{1} d \theta_{2} \\
& I_{8}=-\int_{\left|\theta_{1}\right| \geqslant T} \int_{-T}^{T} \exp \left\{-i \theta_{1} u-i \theta_{2} v-2^{-1}\left(\theta_{1}^{2}+\theta_{2}^{2}\right)\right\} d \theta_{1} d \theta_{2} .
\end{aligned}
$$

В силу леммы 3 , интеграл $I_{1} \rightarrow 0$ при любом фиксированном $T$. Очевидно, что

$$
\left|I_{7}\right| \leqslant \int_{-\infty}^{\infty} \exp \left\{-\theta_{1}^{2} / 2\right\} d \theta_{1} \int_{\left|\theta_{2}\right| \geqslant T} \exp \left\{-\theta_{2}^{2} / 2\right\} d \theta_{2}
$$

и этот интеграл можно сделать сколь угодно малым выбором достаточно большого $T$. Аналогичная оценка справедлива и для интеграла $I_{8}$.

Рассмотрим $I_{2}$. При выполнении условий теоремы 1 в области $M_{2}$ справедлива лемма 5, поэтому

$$
\left|I_{2}\right| \leqslant \int_{-\infty}^{\infty} \exp \left\{-C_{3} \theta_{1}^{2}\right\} d \theta_{1} \int_{\left|\theta_{2}\right|>T} \exp \left\{-C_{4} \theta_{2}^{2}\right\} d \theta_{2},
$$

здесь и далее символы $C_{3}, C_{4}, \ldots$ обозначают некоторые положительные постоянные. Легко видеть отсюда, что $\left|I_{2}\right|$ можно сделать сколь угодно малым выбором достаточно большого $T$. Пусть выполнены условия теоремы 2. Тогда разобьем область $M_{2}$ на три части:

$$
\begin{aligned}
& M_{2}^{(1)}=\left\{\left(\theta_{1}, \theta_{2}\right):\left|\theta_{1}\right| \leqslant \delta(N / n) \sqrt{N \mathrm{D} \mu_{1}(t)}, T<\left|\theta_{2}\right| \leqslant \delta(N / n)^{2} \sqrt{N \mathrm{D} \nu_{1}(t)}\right\}, \\
& M_{2}^{(2)}=\left\{\left(\theta_{1}, \theta_{2}\right): \delta(N / n) \sqrt{N \mathrm{D} \mu_{1}(t)}<\left|\theta_{1}\right| \leqslant \delta \sqrt{N \mathrm{D} \mu_{1}(t)}, T<\left|\theta_{2}\right| \leqslant \delta(N / n)^{2} \sqrt{N \mathrm{D} \nu_{1}(t)}\right\}, \\
& M_{2}^{(3)}=\left\{\left(\theta_{1}, \theta_{2}\right):\left|\theta_{1}\right| \leqslant \delta \sqrt{N \mathrm{D} \mu_{1}(t)}, \delta(N / n)^{2} \sqrt{N \mathrm{D} \nu_{1}(t)}<\left|\theta_{2}\right| \leqslant \delta \sqrt{N \mathrm{D} \nu_{1}(t)}\right\} .
\end{aligned}
$$

Обозначим соответствующие этим областям части интеграла $I_{2}$ через $I_{2}^{(1)}, I_{2}^{(2)}$, $I_{2}^{(3)}$. Применяя для оценки $I_{2}^{(1)}$ лемму 5 , получаем оценку, аналогичную (11). Из леммы 9 и (4) следует, что

$$
\left|I_{2}^{(2)}\right| \leqslant C_{5}(N / \sqrt{n}) \int_{N m^{t / 2} / \sqrt{n}} \exp \left\{-C_{6} \theta_{1} m^{t} N^{2} / n\right\} d \theta_{1} \leqslant C_{7} \sqrt{n} \exp \left\{-C_{6} N \sqrt{m^{t} / n}\right\} .
$$

Отсюда и из условия

$$
\sqrt{n} \leqslant(N / \sqrt{n})\left(N \sqrt{m^{t} / n}\right)^{L} \leqslant\left(N \sqrt{m^{t} / n}\right)^{2 L}
$$

получаем, что $I_{2}^{(2)} \rightarrow 0$. Применяя для оценки $I_{2}^{(3)}$ лемму 10 и (4), находим, что

$$
\begin{aligned}
\left|I_{2}^{(3)}\right| & \leqslant \iint_{M_{2}^{(3)}} \exp \left\{-C_{7} N \sqrt{\left|\theta_{2}\right| / \sqrt{N D \nu_{1}(t)}}\right\} d \theta_{1} d \theta_{2} \\
& \leqslant C_{8} \sqrt{n m^{t}} \int_{N / \sqrt{n}}^{\infty} \exp \left\{-C_{8} \sqrt{N^{3} \theta_{2} / n \sqrt{n}}\right\} d \theta_{2},
\end{aligned}
$$


поэтому с помощью (12) получаем, что

$$
\left|I_{2}^{(3)}\right|<C_{8}(N / \sqrt{n})^{L} \exp \left\{-N^{2} / n\right\} \rightarrow 0 .
$$

Таким образом, в условиях теоремы $2 I_{2} \rightarrow 0$.

Пусть выполнены условия теоремы 4. Разобьем область $M_{2}$ на следующие части:

$$
\begin{aligned}
& M_{2}^{(1)}=\left\{\left(\theta_{1}, \theta_{2}\right):\left|\theta_{1}\right| \leqslant \delta \sqrt{N \mathrm{D} \mu_{1}(t)} / t, T<\left|\theta_{2}\right| \leqslant \delta N \sqrt{N \mathrm{D} \nu_{1}(t)} / n t\right\}, \\
& M_{2}^{(2)}=\left\{\left(\theta_{1}, \theta_{2}\right): \delta \sqrt{N \mathrm{D} \mu_{1}(t)} / t<\left|\theta_{1}\right| \leqslant \delta \sqrt{N \mathrm{D} \mu_{1}(t)}, T<\left|\theta_{2}\right| \leqslant \delta N \sqrt{N \mathrm{D} \nu_{1}(t)} / n t\right\}, \\
& M_{2}^{(3)}=\left\{\left(\theta_{1}, \theta_{2}\right):\left|\theta_{1}\right| \leqslant \delta \sqrt{N \mathrm{D} \mu_{1}(t)}, \delta N \sqrt{N \mathrm{D} \nu_{1}(t)} / n t<\left|\theta_{2}\right| \leqslant \delta \sqrt{N \mathrm{D} \nu_{1}(t)}\right\},
\end{aligned}
$$

и снова обозначим $I_{2}^{(1)}, I_{2}^{(2)}, I_{2}^{(3)}$ соответствующие части $I_{2}$. Интеграл $I_{2}^{(1)}$ с помощью леммы 5 оценивается аналогично предыдущему. Нетрудно видеть, что в области $M_{2}^{(2)}$ можно использовать лемму 9 , поэтому с помощью (4) получаем, что

$$
\left|I_{2}^{(2)}\right| \leqslant C_{9}(N / \sqrt{n}) \int_{\sqrt{N / t}}^{\infty} \exp \left\{-C_{10} N \theta_{1} /(t \sqrt{N t})\right\} d \theta_{1}<C_{11} t^{3 / 2} e^{-t} \rightarrow 0 .
$$

Из леммы $10,(4)$ и условий $n / N<(N / \sqrt{n})^{L}, t=o(N / n)$ следует, что

$$
\left|I_{2}^{(3)}\right| \leqslant C_{12} \sqrt{N t} \int_{N / \sqrt{n}}^{\infty} \exp \left\{-C_{13} N \theta_{2} / \sqrt{n}\right\} d \theta_{2}<C_{12}(N / \sqrt{n})^{L} \exp \left\{-C_{14} N^{2} / \sqrt{n}\right\} \rightarrow 0
$$

Оценим $I_{3}$. При выполнении условий теоремы 1 в области $M_{3}$ выполняется лемма 5 , поэтому $I_{3}$ оценивается аналогично $I_{2}$. В условиях теоремы 2 разобьем $M_{3}$ на две части:

$$
\begin{aligned}
& M_{3}^{(1)}=\left\{\left(\theta_{1}, \theta_{2}\right): T \leqslant\left|\theta_{1}\right| \leqslant \delta(N / n) \sqrt{N \mathrm{D} \mu_{1}(t)},\left|\theta_{2}\right| \leqslant T\right\}, \\
& M_{3}^{(2)}=\left\{\left(\theta_{1}, \theta_{2}\right): \operatorname{de}(N / n) \sqrt{N \mathbf{D} \mu_{1}(t)}<\left|\theta_{1}\right| \leqslant \sqrt{N \mathbf{D} \mu_{1}(t)},\left|\theta_{2}\right| \leqslant T\right\},
\end{aligned}
$$

тогда, соответственно, $I_{3}=I_{3}^{(1)}+I_{3}^{(2)}$. Интеграл $I_{3}^{(1)}$ с помощью леммы 5 оценивается аналогично $I_{2}^{(1)}$, а соотношение $I_{3}^{(2)} \rightarrow 0$ доказывается с помощью леммы 9 , как и в случае $I_{2}^{(2)}$. При выполнении условий теоремы 4 положим

$$
\begin{aligned}
& M_{3}^{(1)}=\left\{\left(\theta_{1}, \theta_{2}\right): T \leqslant\left|\theta_{1}\right| \leqslant \delta \sqrt{N \mathrm{D} \mu_{1}(t)} / t,\left|\theta_{2}\right| \leqslant T\right\}, \\
& M_{3}^{(2)}=\left\{\left(\theta_{1}, \theta_{2}\right): \delta \sqrt{N \mathbf{D} \mu_{1}(t)} / t<\left|\theta_{1}\right| \leqslant \sqrt{N \mathbf{D} \mu_{1}(t)},\left|\theta_{2}\right| \leqslant T\right\} .
\end{aligned}
$$

В области $M_{3}^{(1)}$ интеграл $I_{3}$ оценивается с помощью леммы 5 аналогично предыдущему, а в $M_{3}^{(2)}$, как и в случае $I_{2}^{(2)}$, применяется лемма 9.

Рассмотрим $I_{4}$. Ясно, что для оценки этого интеграла можно использовать лемму 9. Тогда при выполнении условий теоремы 1 из (4) следует, что

$$
\left|I_{4}\right| \leqslant \iint_{M_{4}} \exp \left\{-C_{15} m^{t} N^{2} / n\right\} d \theta_{1} d \theta_{2} \leqslant C_{16} \sqrt{m} N m^{t-1} \exp \left\{-C_{17} N m^{t-1}\right\} \rightarrow 0 .
$$

Для теоремы 2 аналогично получаем, что

$$
\left|I_{4}\right| \leqslant C_{18} n m^{t} \exp \left\{-C_{19} m^{t} N^{2} / n\right\} \text {. }
$$


Учитывая, что $n m^{t}<\left(N \sqrt{m^{t} / n}\right)^{2 L+2}$, находим, что $I_{4} \rightarrow 0$. В случае теоремы 4 приходим к оценке

$$
\left|I_{4}\right| \leqslant C_{20} \sqrt{N n} \exp \left\{-C_{21} N^{2} / n\right\}
$$

и, учитывая неравенство $\sqrt{n}<(N / \sqrt{n})^{L+1}$, получаем, что $I_{4} \rightarrow 0$ и в этом случае.

Оценим интеграл $I_{5}$. Пусть выполнены условия теоремы 1 . Тогда в области $M_{5}$ действует лемма 7. Отсюда

$$
\begin{aligned}
\left|I_{5}\right| & \leqslant \iint_{M_{5}} \exp \left\{-C_{22} \theta_{2}^{2}\right\} d \theta_{1} d \theta_{2} \leqslant C_{23} \sqrt{N D \mu_{1}(t)} \int_{\left|\theta_{2}\right|>4 \sqrt{N m^{t}}} \exp \left\{-C_{22} \theta_{2}^{2}\right\} d \theta_{2} \\
& \leqslant C_{24} \int_{\sqrt{N m^{t}}}^{\infty} \theta \exp \left\{-C_{22} \theta^{2}\right\} d \theta
\end{aligned}
$$

Поскольку $\sqrt{N m^{t}} \rightarrow \infty$, получаем, что $I_{5} \rightarrow 0$. В условиях теоремы 2 разобьем $M_{5}$ на две части:

$M_{5}^{(1)}=\left\{\left(\theta_{1}, \theta_{2}\right): \delta \sqrt{N \mathbf{D} \mu_{1}(t)}<\left|\theta_{1}\right| \leqslant \pi \sqrt{N \mathbf{D} \mu_{1}(t)} / d, \sqrt{N m^{t}} \leqslant\left|\theta_{2}\right| \leqslant \delta(N / n)^{2} \sqrt{N \mathbf{D} \nu_{1}(t)}\right\}$ $M_{5}^{(2)}=\left\{\left(\theta_{1}, \theta_{2}\right): \delta \sqrt{N \mathbf{D} \mu_{1}(t)}<\left|\theta_{1}\right| \leqslant \pi \sqrt{N \mathbf{D} \mu_{1}(t)} / d, \delta(N / n)^{2} \sqrt{N \mathbf{D} \nu_{1}(t)} \leqslant \delta \sqrt{N \mathbf{D} \nu_{1}(t)}\right\}$

и обозначим соответствующие интегралы $I_{5}^{(1)}, I_{5}^{(2)}$. Используя для оценки $I_{5}^{(1)}$ лемму 7 и (4), получаем, что

$$
\left|I_{5}^{(1)}\right| \leqslant C_{25} \sqrt{N \mathrm{D} \mu_{1}(t)} \int_{\sqrt{N m^{t}}}^{\infty} \exp \left\{-C_{22} \theta_{2}^{2}\right\} d \theta_{2} \leqslant C_{26} \sqrt{n / N} \exp \left\{-C_{22} N m^{t}\right\} .
$$

Заметим, что $\sqrt{n / N}<\left(N \sqrt{m^{t} / n}\right)^{L / 2}, N m^{t}>N^{2} m^{t} / n$, поэтому $I_{5}^{(1)} \rightarrow 0$. Легко видеть, что оценка $I_{5}^{(2)}$ аналогична оценке $I_{2}^{(3)}$, следовательно, $I_{5} \rightarrow 0$.

Пусть выполнены условия теоремы 4. Применяя лемму 9 и (4), находим, что

$$
\left|I_{5}\right| \leqslant C_{27} t^{3 / 2} \sqrt{n N} \exp \left\{-C_{28} N / t\right\} \leqslant C_{27}\left(n^{2} / N\right) \exp \left\{-C_{28} N^{2} / n\right\}
$$

и, поскольку $\sqrt{n}<(N / \sqrt{n})^{L+1}$, получаем соотношение $I_{5} \rightarrow 0$.

Нам осталось оценить $I_{6}$. Используя лемму 8 получаем, что

$$
\left|I_{6}\right| \leqslant C_{29} N \sqrt{\mathbf{D} \mu_{1}(t) \mathbf{D} \nu_{1}(t)} q_{0}^{N} .
$$

Оценивая дисперсии с помощью (4), находим отсюда, что $I_{6} \rightarrow 0$. Лемма 11 доказана.

Таким же способом можно получить следующее утверждение.

Лемма 12. При выполнении условий теоремъ 3

$$
\begin{aligned}
& \sup _{k, s} \mid N d^{-2} \sqrt{\mathbf{D} \mu_{1}(t) \mathbf{D} \nu_{1}(t)\left(1-\rho^{2}\right)} \mathbf{P}\left\{\mu_{N}(t)=k, \nu_{N}(t)=s\right\} \\
&-(2 \pi)^{-1} \exp \left\{-\left(2\left(1-\rho^{2}\right)\right)^{-1}\left(u^{2}-2 \rho u v+v^{2}\right)\right\} \mid \rightarrow 0
\end{aligned}
$$

¿de

$$
u=\left(k-N \mathrm{M} \mu_{1}(t)\right) / \sqrt{N \mathrm{D} \mu_{1}(t)}, \quad v=\left(s-N \mathrm{M} \nu_{1}(t)\right) / \sqrt{N \mathrm{D} \nu_{1}(t)},
$$

а величина $\rho$ определена в теореме 3. 
Введем величину $z(N, n)$ следующим образом:

$$
z(N, n)= \begin{cases}\sqrt{(N+n) B_{\lambda} m^{t-1}} & \text { в условиях теорем } 1,2 ; \\ \sqrt{H n B_{\lambda}} & \text { в условиях теоремы } 3 ; \\ \sqrt{N D \mu_{1}(t)} & \text { в условиях теоремы } 4\end{cases}
$$

Лемма 13. Пусть выполнены условия одной из теорем 1-4. Тогда равномерно относительно $k$, для которых $y=\left(k-N m^{t}\right) / z(N, n)$ лежит в любом конечном интервале,

$$
\mathbf{P}\left\{\mu_{N}(t)=k \mid \nu=N+n\right\}=\frac{d(1+o(1))}{\sqrt{2 \pi} z(N, n)} e^{-y^{2} / 2} .
$$

Доказательство. Справедливо равенство

$$
\begin{aligned}
\mathbf{P}\left\{\mu_{N}(t)\right. & \left.=k \mid \nu_{N}=N+n\right\} \\
& =\sum_{s=N}^{N+n-k} \mathbf{P}\left\{\mu_{N}(t)=k, \nu_{N}(t)=s\right\} \mathbf{P}\left\{\nu_{k}=N+n-s\right\} / \mathbf{P}\left\{\nu_{N}=N+n\right\} .
\end{aligned}
$$

Пусть выполнены условия теоремы 1 или теоремы 2. Разобьем область суммирования на две части:

$$
\begin{aligned}
& M_{1}=\left\{s:(N+n)\left(1-m^{t}\right)-t \sqrt{(N+n) m^{t}} \leqslant s \leqslant(N+n)\left(1-m^{t}\right)+t \sqrt{(N+n) m^{t}}\right\} \\
& M_{2}=\left\{s: N \leqslant s \leqslant N+n-k, s \notin M_{1}\right\} .
\end{aligned}
$$

Нетрудно проверить, что при $s \in M_{1}$ к вероятности $\mathbf{P}\left\{\mu_{N}(t)=k, \nu_{N}(t)=s\right\}$ можно применить лемму 11 , при этом $v=o(1), u=y(1+o(1))$, следовательно,

$$
\mathbf{P}\left\{\mu_{N}(t)=k, \nu_{N}(t)=s\right\}=\frac{d^{2}(1+o(1))}{2 \pi N \sqrt{\mathbf{D} \mu_{1}(t) \mathbf{D} \nu_{1}(t)}} e^{-y^{2} / 2} .
$$

Понятно, что случайная величина $\nu_{k}$ является суммой $k$ независимых случайных величин с распределением $\nu_{1}$. Заметим также, что

$$
\begin{aligned}
& \left((N+n) m^{t}-t \sqrt{(N+n) m^{t}}-k(N+n) N^{-1}\right) / \sqrt{k \mathbf{D} \nu_{1}(t)} \rightarrow-\infty \\
& \left((N+n) m^{t}-t \sqrt{(N+n) m^{t}}+k(N+n) N^{-1}\right) / \sqrt{k \mathbf{D} \nu_{1}(t)} \rightarrow \infty
\end{aligned}
$$

Повторяя дословно доказательство леммы 2.3.1 из [3], нетрудно показать, что для суммы $\nu_{k}$ выполняется центральная предельная теорема, поэтому, учитывая (15), получаем, что

$$
\sum_{s \in M_{2}} \mathbf{P}\left\{\nu_{k}=N+n-s\right\}=1+o(1) .
$$

Согласно лемме 2.3.2 [3]

$$
\mathbf{P}\left\{\nu_{N}=N+n\right\}=\frac{d N(1+o(1))}{(N+n)^{3 / 2} \sqrt{2 \pi B_{\lambda}}},
$$


поэтому из (13), (14) и (16) получаем, что

$$
\sum_{s \in M_{1}} \mathbf{P}\left\{\mu_{N}(t)=k, \nu_{N}(t)=s\right\} \frac{\mathbf{P}\left\{\nu_{k}=N+n-s\right\}}{\mathbf{P}\left\{\nu_{N}=N+n\right\}}=\frac{d(1+o(1))}{\sqrt{2 \pi(N+n) B_{\lambda} m^{t-1}}} e^{-y^{2} / 2}
$$

Рассуждая аналогично, нетрудно убедиться, что соответствующая сумма в области $M_{2}$ стремится к нулю.

Пусть выполнены условия теоремы 3. Тогда область суммирования в (13) разобъем на такие две части:

$$
\begin{aligned}
& M_{1}=\left\{s:(N+n)\left(1-m^{t}\right)-A t \sqrt{n m^{t}} \leqslant s \leqslant(N+n)\left(1-m^{t}\right)+A t \sqrt{n m^{t}}\right\}, \\
& M_{2}=\left\{s: N \leqslant s \leqslant N+n-k, s \notin M_{1}\right\},
\end{aligned}
$$

выбор постоянной $A$ осуществляется ниже. Применяя к $\mathbf{P}\left\{\mu_{N}(t)=k, \nu_{N}(t)=s\right\}$ лемму 12 и проведя необходимые преобразования, получаем, аналогично предыдущему, что при достаточно больших $A$ сумма

$$
\sum_{s \in M_{1}} \mathbf{P}\left\{\mu_{N}(t)=k, \nu_{N}(t)=s\right\} \mathbf{P}\left\{\nu_{k}(t)=N+n-s\right\} / \mathbf{P}\left\{\nu_{k}=N+n\right\}
$$

сколь угодно мало отличается от

$$
\frac{d(1+o(1))}{\sqrt{2 \pi \operatorname{HnB}_{\lambda}}} e^{-y^{2} / 2}
$$

а вторая часть суммы стремится к нулю. Таким же образом устанавливается справедливость леммы 13 и в условиях теоремы 4.

Лемма 14. Пусть выполнены условия теоремы 5. Тогда для любого фиксированного $x$

$$
\mathbf{P}\left\{\frac{\mu_{N}(t)-N m^{t}}{\sqrt{\mathbf{D} \mu_{N}(t)}}<x \mid \nu_{N}=N+n\right\} \rightarrow \frac{1}{\sqrt{2 \pi}} \int_{-\infty}^{x} e^{-y^{2} / 2} d y .
$$

Доказательство. Для произвольных $x_{1}<x_{2}$ положим

$$
k_{1}=N m^{t}+x_{1} \sqrt{\mathbf{D} \mu_{N}(t)}, \quad k_{2}=N m^{t}+x_{2} \sqrt{\mathbf{D} \mu_{N}(t)} .
$$

Легко видеть, что

$$
\begin{aligned}
& \mathbf{P}\left\{x_{1} \leqslant\left(\mu_{N}(t)-N m^{t}\right) / \sqrt{\mathbf{D} \mu_{N}(t)} \leqslant x_{2} \mid \nu_{N}=N+n\right\} \\
& =\sum_{k_{1} \leqslant k \leqslant k_{2}} \mathbf{P}\left\{\mu_{N}(t)=k \mid \nu_{N}=N+n\right\} \\
& =\sum_{k_{1} \leqslant k \leqslant k_{2}} \sum_{s=N}^{N+n-k} \mathbf{P}\left\{\mu_{N}(t)=k, \nu_{N}(t)=s\right\} \mathbf{P}\left\{\nu_{k}=N+n-s\right\} / \mathbf{P}\left\{\nu_{N}=N+n\right\} .
\end{aligned}
$$

Положим $\gamma=\varepsilon \sqrt{n} / N$, где положительная постоянная $\varepsilon$ будет выбрана позднее. Разобьем область изменения индекса $s$ в сумме (18) на две части:

$$
\begin{aligned}
& M_{1}=\{s: N<s \leqslant n \gamma\}, \\
& M_{2}=\{s: n \gamma<s \leqslant N t n-k\},
\end{aligned}
$$


и обозначим $S_{1}$ и $S_{2}$ соответствующие суммы. Тогда

$$
\mathbf{P}\left\{x_{1} \leqslant \frac{\mu_{N}(t)-N m^{t}}{\sqrt{\mathbf{D} \mu_{N}(t)}} \leqslant x_{2} \mid \nu_{N}=N+n\right\}=S_{1}+S_{2} .
$$

Применяя лемму 2.3.2 [3] и (17), нетрудно получить, что при $s \in M_{1}$

$$
\mathbf{P}\left\{\nu_{k}=N+n-s\right\} / \mathbf{P}\left\{\nu_{N}=N+n\right\}=1+o(1),
$$

поэтому

$$
\begin{aligned}
S_{1} & =(1+o(1)) \sum_{k_{1} \leqslant k \leqslant k_{2}} \sum_{s \in M_{1}} \mathbf{P}\left\{\mu_{N}(t)=k, \nu_{N}(t)=s\right\} \\
& =\left(\mathbf{P}\left\{k_{1} \leqslant \mu_{N}(t) \leqslant k_{2}\right\}-\mathbf{P}\left\{k_{1} \leqslant \mu_{N}(t) \leqslant k_{2}, \nu_{N}(t)>n \gamma\right\}\right)(1+o(1)) .
\end{aligned}
$$

Используя явные выражения первых трех моментов случайной величины $\mu_{1}(t)$, нетрудно проверить; что для суммы $\mu_{N}(t)$, образованной $N$ независимыми слагаемыми с распределением $\mu_{1}(t)$, выполнены условия Ляпунова, поэтому при достаточно больших $N, n$ и достаточно малом $\varepsilon$ вероятность $\mathbf{P}\left\{k_{1} \leqslant \mu_{N}(t) \leqslant k_{2}\right\}$ сколь угодно мало отличается от

$$
\frac{1}{\sqrt{2 \pi}} \int_{x_{1}}^{x_{2}} e^{-y^{2} / 2} d y
$$

С помощью неравенства Чебышева и (4) нетрудно показать, что

$$
\mathbf{P}\left\{k_{1} \leqslant \mu_{N}(t) \leqslant k_{2}, \nu_{N}(t)>n \gamma\right\} \leqslant \mathbf{P}\left\{\mu_{N}(t)>n \dot{\gamma}\right\} \rightarrow 0,
$$

поэтому из (20) следует, что

$$
S_{1}=\frac{1}{\sqrt{2 \pi}} \int_{x_{1}}^{x_{2}} e^{-y^{2} / 2} d y+o(1) .
$$

Для оценки $S_{2}$ рассмотрим сначала $\mathbf{P}\left\{\nu_{k}=N+n-s\right\}$. По лемме 2.1.3 из [4]

$$
\mathbf{P}\left\{\nu_{k}=N+n-s\right\}=\frac{k}{N+n-s} \mathbf{P}\left\{\xi_{1}+\ldots+\xi_{N+n-s}=N_{n}-s-k\right\},
$$

где $\xi_{1}, \ldots, \xi_{N+n-s}-$ независимые случайные величины с распределением (1). Используя теорему 7.13.16 книги [11], нетрудно получить, что

$$
\mathbf{P}\left\{\nu_{k}=N+n-s\right\} \leqslant C / N^{2},
$$

где $C$ - некоторая положительная постоянная. Отсюда и из (17) получаем, что

$$
S_{2} \leqslant C N^{-5 / 2} \mathbf{P}\left\{k_{1} \leqslant \mu_{N}(t) \leqslant k_{2}, n \gamma<\nu_{N}(t) \leqslant N+n-s\right\}
$$

и, опять применяя неравенство Чебышева, находим, что

$$
S_{2} \leqslant C N^{-5 / 2} \mathbf{P}\left\{\nu_{N}(t)>\gamma n\right\} \leqslant C t / N^{5 / 2} \varepsilon^{2} n \rightarrow 0 .
$$

Отсюда и из (19), (21) получаем утверждение леммы 14.

Теперь, используя лемму 2, легко установить справедливость теорем 1-6. Теоремы 1-4 следуют из леммы 13 , а теорема 5 вытекает из леммы 14 . Доказательство теоремы 6 почти дословно повторяет доказательство теоремы 3.2 работы [1]. 


\section{Список литературы}

1. Калугин И. Б. Один класс случайных отображений. Труды МИАН CCCP (1986) 177, 75-104.

2. Pavlov $\mathrm{Yu}$. L. On the distributions of the number of vertices in strata of a random forest. In: Proc. 1st World Congress Bernoulli Soc., V.1: Probability and Applications. VNU Science Press. Utrecht, 1987, pp. 239-241.

3. Павлов Ю. Л. Случайнъе леса. Карельский НЦ РАН, Петрозаводск, 1996.

4. Колчин В. Ф. Случайные отображения. Наука, Москва, 1984.

5. Egorova I. A. The distribution of vertices in strata of plane planted forest. In: Probabilistic Methods in Discrete Mathematics: Proc. 4th Intern. Petrozavodsk Conf. VSP, Utrecht, 1997, pp. 179-188.

6. Чеплюкова И. А. Предельные распределения числа вершин в слоях случайного леса. Дискретная математика (1997) 9, №4, 150-157.

7. Чеплюкова И. А. Возникновение гигантского дерева в случайном лесе. Дискретная математика (1998) 10, №1, 111-126.

8. Meir A., Moon J. W. On the altitude of nodes in random trees. Canad. J. Math. (1978) 30, 997-1015.

9. Дрмота М. Распределения высоты листьев корневых деревьев. Дискретнал матемаmuка (1994) 6, №1, 67-82.

10. Севастьянов Б. А. Ветөящиеся процессы. Наука, Москва, 1971.

11. Ибрагимов И. А., Линник Ю. В. Независимые и стационарно связанные величины. Наука, Москва, 1965.

Статья поступила 06.12.1998. 